\title{
Privacy Protection for a Secure u-City Life
}

\author{
Changjin Lee, Bong Gyou Lee, and Youngil Kong \\ Graduate School of Information, Yonsei University \\ 134 Shinchondong, Seoul 120-749, Korea \\ \{cjlee, bglee, okay777\} @yonsei.ac.kr
}

\begin{abstract}
Recently, projects to construct an innovative brand-new city, termed a u-City (ubiquitous City), are being carried out in many countries. The u-City is a future-oriented city which combines ubiquitous information services with city. It has emerged as an alternative for improving the quality of life for human beings and reaching the balanced development among cities. Although a number of privacy problems are anticipated, there are very few researches on the protection of privacy in the u-City. In this study, we describe the privacy guidelines for modern cities as well as the privacy issues in the u-City, and provide suggestions for privacy protection to establish a safe u-City.
\end{abstract}

Keywords: Ubiquitous Computing, u-City, Personal Information, Privacy Protection.

\section{Introduction}

Ubiquitous computing technologies have been applied to many fields including public service, education, health, and transportation. These technologies are being combined with city, and then make it possible to build up a new city, u-City. The u-City is a sophisticated and intelligent city where information can be easily exchanged among people, objects and environments inside the city based on the ubiquitous network. The u-City is aimed to improve the quality of people's life in the city by building up a human-centered city, and to accomplish balanced development among cities by specializing their own strength [1].

However, in the u-City, private information becomes readily available and as such, privacy issues may become a serious problem. Since most projects to construct the $\mathrm{u}$-City are in the very early stage, there is few research on privacy issues in the u-City. In this research, therefore, we look into various issues in privacy violation which should be considered in the process of u-City planning, and then make suggestions for a safer u-City life.

We briefly explain the features of u-City in chapter 2 . The privacy issues in the $\mathrm{u}$-City are described in chapter 3 . In chapter 4, we provide some suggestions for privacy protection in the u-City. Conclusion and future direction of a secure u-City are presented in chapter 5 . 


\section{What Is the u-City?}

The u-City is different from the Digital Cities which are currently being constructed in many countries over the world. The Digital City collects and organizes the digital information of the corresponding physical city, and provides a public information space for people living in and visiting the city to interact with each other [2].

However, the u-City is a complex of $\mathrm{u}$-Home, u-Work, u-Transportation, $\mathrm{u}$-Environment, u-Health, u-Education, and u-Government based on ubiquitous computing technologies such as USN (Ubiquitous Sensor Network), RFID (Radio Frequency Identification), and FTTH (Fiber To The Home) as shown in Figure 1 [3].

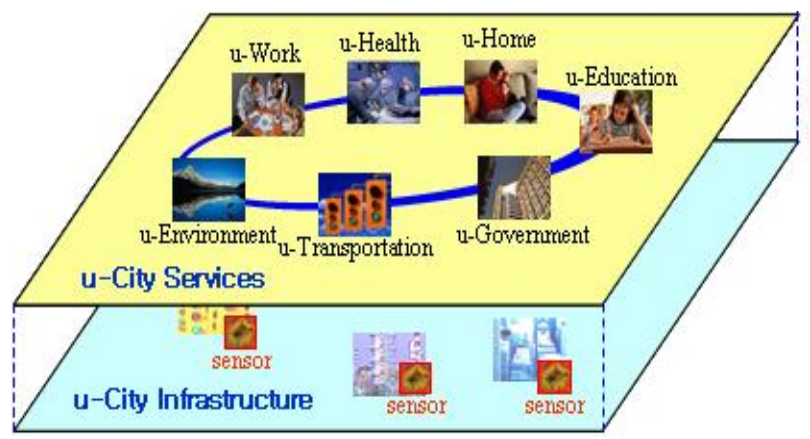

Fig. 1. The Concept of u-City

The differences between a modern city, Digital City and u-City are summarized in Table 1.

Table 1. Modern City vs. Digital City vs. u-City

\begin{tabular}{|c|c|c|}
\hline Modern city & Digital city & u-City \\
\hline Distance centric & Information centric & Human centric \\
\hline Spatial, temporal limitation & $\begin{array}{l}\text { Spatial, temporal limitation } \\
\text { relieved }\end{array}$ & $\begin{array}{l}\text { No spatial, temporal } \\
\text { limitation }\end{array}$ \\
\hline Real space & $\begin{array}{l}\text { Division of real and virtual } \\
\text { space }\end{array}$ & $\begin{array}{l}\text { Convergence of real and } \\
\text { virtual space }\end{array}$ \\
\hline $\begin{array}{l}\text { Environment } \\
\text { and energy problem }\end{array}$ & $\begin{array}{l}\text { Environment and energy } \\
\text { problem }\end{array}$ & Environment friendly city \\
\hline $\begin{array}{l}\text { Limitation } \\
\text { on accommodating } \\
\text { new function }\end{array}$ & $\begin{array}{l}\text { Limitation } \\
\text { on accommodating } \\
\text { new function }\end{array}$ & Efficient city management \\
\hline Producer centric & Producer centric & Consumer centric \\
\hline $\begin{array}{l}\text { Unbalanced development } \\
\text { among region }\end{array}$ & $\begin{array}{l}\text { Unbalanced development } \\
\text { among region }\end{array}$ & $\begin{array}{l}\text { Balanced development } \\
\text { among region }\end{array}$ \\
\hline
\end{tabular}




\section{Privacy Issues in the u-City}

\subsection{Guidelines for Privacy Protection by International Organizations}

Traditionally, 'privacy' is defined in various ways. It does not have specific or definite legal connotation [4] and as such the concept of 'privacy' can be too broad. Thus, this paper will focus on the information privacy related to protection of personal information. 'Privacy' means the right or obligation of an individual or entities to collect, use and disclose personal information [5], and personal information refers to any type of information that identify or can identify an individual or an entity [6].

So far, international efforts have focused on the protection of privacy with regards to collecting, processing and distribution of personal information. OECD was first to provide guidelines on the protection of privacy (Guidelines Governing the Protection of Privacy and Transborder Flows of Personal Data) in 1980 as the development of electronic data processing technique made mass data transfer possible [7]. Since then, development of new guidelines on privacy has followed in the UN, EU and APEC with a view to providing their members with references on privacy protection.

The OECD Guidelines Governing the Protection of Privacy and Transborder Flows of Personal Data is composed of 8 principles ; 1) Collection Limitation Principle, 2) Data Quality Principle, 3) Purpose Specification Principle, 4) Use Limitation Principle, 5) Security Safeguard Principle, 6) Openness Principle, 7) Individual Participation Principle, 8) Accountability Principle. The Guidelines provide recommendations to the member countries although no binding force exists.

The OECD Guidelines had a direct effect on the privacy guidelines or rules developed later such as the UN Guidelines for the Regulation of Computerized Personal Data Files (1990) [8], EU Directive on the Protection of Individuals with regard to the Processing of Personal Data and on the Free Movement of Such Data (1995) [9] and APEC Information Privacy Principles (2004) [10]. Thus, these guidelines accommodate most of the 8 principles suggested in the OECD Guidelines and build upon the OECD Guidelines by adding new text relevant to each situation.

The OECD Guidelines function as the basic principle for privacy protection and can be seen in the Privacy Laws of many countries such as England, Sweden, Canada, Hong Kong, Australia, and New Zealand. UN Guidelines recommend that member countries reference the guidelines when making privacy laws or procedures of their countries. The EU Directive provides principles and standards for processing personal data and the Directive has been adopted by the domestic laws of its member countries.

Also, in October 2004, APEC adopted guidelines on privacy in October 2004. Based on the OECD Guidelines, the APEC "Information Privacy Principles" added 3 new principles; Preventing Harm, Notice, Choice.

\subsection{Privacy Issues in the u-City}

The u-City is a new space where physical space and electronic space are integrated into one, quite different from the concept of digital city, in which a modern city or a physical space is simply being reflected or applied to an electronic space. In other words, in the u-City, computers, which are interconnected by ubiquitous networks, are embedded in human beings, objects, and environments and offer further convenience 
for residents. However, the side-effect is that private information becomes readily available and as such, privacy issues may become a serious problem. Privacy issues that may appear in the u-City will be reviewed based on the OCED Principles for the Protection of Privacy.

First, in the u-City, there is a high possibility for the Collection Limitation Principle to be violated. One of the key technologies in the u-City is sensing and tag technologies such as RFID, which enable large amounts of private information to be collected in real-time regardless of one's own will. For example, by analyzing RFID information of a purchased item by an individual, one can speculate about the individual's patterns of consumption, social status, current physical location, or health-related information regardless of the individual's will. Thus, personal data can be collected through illegal means without the consent of the subject. Furthermore, under the environment of the u-City, it may sometimes be impossible to notify in advance and ask for consent from the subject for collecting data. As a result, the uCity is highly vulnerable to infringement of the Collection Limitation Principle.

Second, in the u-City, there is also a high possibility of violating the Data Quality Principle. As previously explained in the Collection Limitation Principle, in the u-City, immense amounts of personal data-both necessary and unnecessary - are collected in real-time through computers that are embedded in every object. These data may not all be relevant to the purpose of use, causing inconsistencies in data. This problem can raise problems with the quality of personal data.

Third, the u-City can raise problems with the Purpose Specification Principle. For example, in addition to purpose-specified personal data, other data such as individual location can be collected by identifying the course of movement of an individual through using a telematic service. Also, using RFID chips, sensitive information such as medical conditions or patterns of consumption can also be collected.

Fourth, in terms of the Use Limitation Principle, the u-City is not much different from a modern city since both are exposed to the same danger of using personal data for purposes other than those specified in accordance with the Purpose Specification Principle. The only difference between the two cities is the amount of personal data collected.

Fifth, compared with modern cities, the u-City has a higher possibility of violation of the Security Safeguard Principle. In the u-City, computers are embedded in every device and as each device is interconnected, more personal data is exposed to larger number of people than in modern city. Thus, the danger of personal data being used by malicious users such as hackers increases in the u-City, causing such risks as unauthorized access, destruction, use, modification or disclosure of data.

Sixth, the Openness Principle is also easily violated in the u-City compared to the modern city. In modern cities, which are internet-based, personal data is collected openly mainly through websites or via off-line. Most websites or data controllers notify their subscribers about the policies of protection of private information. However, in the u-City, personal data can often be collected without previous consent or recognition of the data subject and the means to notify the subscribers of policies on private information protection are limited. Thus, in the u-City, the Openness Principles are more difficult to implement than in modern cities. 
Table 2. Possibility for Privacy Violation(Modern City vs. u-City)

\begin{tabular}{|c|c|c|c|}
\hline OECD Principle & $\begin{array}{l}\text { Modern } \\
\text { city }\end{array}$ & u-City & Example of privacy invasion in u-City \\
\hline $\begin{array}{l}\text { Collection limitation } \\
\text { principle }\end{array}$ & Low & Very high & $\begin{array}{l}\text { Impossible to notify a subject about } \\
\text { collection of her or his information }\end{array}$ \\
\hline $\begin{array}{l}\text { Data quality } \\
\text { Principle }\end{array}$ & Medium & High & $\begin{array}{l}\text { Discordance among collected private } \\
\text { information }\end{array}$ \\
\hline $\begin{array}{l}\text { Purpose specification } \\
\text { principle }\end{array}$ & Low & High & $\begin{array}{l}\text { Possibility of data analogy without } \\
\text { purpose of collection }\end{array}$ \\
\hline $\begin{array}{l}\text { Use limitation } \\
\text { Principle }\end{array}$ & High & High & $\begin{array}{l}\text { Abuse of private information in } \\
\text { monitoring and pursuing a subject }\end{array}$ \\
\hline $\begin{array}{l}\text { Security safeguard } \\
\text { Principle }\end{array}$ & High & Very high & $\begin{array}{l}\text { Possibility of security violation by } \\
\text { hacking mobile networks }\end{array}$ \\
\hline Openness principle & Medium & High & $\begin{array}{l}\text { Limited means to notify privacy } \\
\text { protection policy to a subject }\end{array}$ \\
\hline $\begin{array}{l}\text { Individual } \\
\text { participation principle }\end{array}$ & Medium & High & Impossible to identify data controller \\
\hline $\begin{array}{l}\text { Accountability } \\
\text { principle }\end{array}$ & Low & High & $\begin{array}{l}\text { Vagueness of data controller and the } \\
\text { location of responsibilities }\end{array}$ \\
\hline
\end{tabular}

Seventh, in the u-City, a large spectrum of personal data-not only one's identification information but also one's current location, current status and so onare collected and processed in real-time basis regardless of one's own will. Therefore, it is impossible in reality for the subject to identify data controllers, who have his or her personal data. Thus, in the u-City, the Individual Participation Principle is difficult to adhere to.

Table 3. Privacy Issues in the u-City

\begin{tabular}{ll}
\hline Category & Privacy issues \\
\hline Information & Information collection without consent by an information subject \\
collection & A breach of notification during the collection of private information \\
& Collection of private information by illegal means such as hacking \\
Information & Collection of private information by illegal monitoring or pressure \\
processing & Outflow of private information by internal data controller \\
& Outflow of private information by an unauthorized person \\
& Outflow of private information by careless manipulation \\
& Outflow of private information by lack of technical measures \\
& Rejection of an information subject' requests to change and delete their \\
& private information \\
& Retention of private information after duration of use is expired \\
Information & Abuse of private information without notice to an information subject \\
dissemination & Information sharing with third party without an information subject's \\
& agreement \\
& Violation of security agreement of private information \\
& Outflow of sensitive information that can put subjects in danger or hurt the \\
& fame \\
& Illegal appropriation of other's ID \\
& Intentional outflow of other people's incorrect information
\end{tabular}


Last, in the u-City, the data controller for specific data is often unclear and difficult to be identified since there are so many channels available to gain access to personal data. Thus, personal data may become poorly managed within the u-City, which in turn, may give rise to infringement of the protection of private information.

Based on the 8 Principles for the Protection of Privacy, Table 2 below summarizes the possibilities of privacy violations that can occur under modern city versus u-City.

Solove (2005) presented the taxonomy of harmful activities on privacy, consisting of information collection, information processing and information dissemination. This taxonomy provides a framework to classify risks with regards to privacy. Thus, privacy issues in u-City can be arranged using the taxonomy as Table 3.

\section{Suggestions for Privacy Protection in the u-City}

In order to achieve privacy-protected and safe u-City life, measures to protect privacy should be considered in various aspects including regulatory and technological framework based on the privacy issues mentioned on the previous chapter.

\subsection{Regulatory and Institutional Suggestions in Building a Safe u-City}

Firstly, in order to strengthen privacy protection in u-City, relevant regulation that reflects the specific characteristics of u-City should be introduced to provide with clear procedures for obtaining consent, providing notification, etc. In u-City, personal activities are exposed to pervasive sensors. As it is sometimes difficult to obtain consent or make notification to the data subject in the u-City environment, the problem of personal information control arises.

However, obtaining consent of the data subject in collecting personal data, specifying the purpose for which personal data are collected, and providing notice regarding the collection and use of personal data is the essential principles that should be observed in order to protect privacy. These basic principles for privacy protection should be ensured in the u-City environment as well. In this context, efforts should be paid to seek appropriate measures to protect privacy in the u-City by providing clear process for obtaining consent and making notification that reflects the circumstances of u-City.

Secondly, regulatory framework should be prepared to protect anonymity of delicate personal data that can be collected in the u-City environment. In the u-City environment, delicate information such as a person's locational and circumstantial information can be induced by combining information collected through sensors, RFID Tags. For example, by analyzing the RFID tag of a medical product which is purchased by an AIDS patient, information that the person is HIV-positive can be induced. When such information is disclosed, the data subject can face the danger of being excluded from society as a result of privacy infringement

Thirdly, regulatory tool to protect personal data from development of new technologies should be devised. In the u-City environment, the continuous evolution of ubiquitous computing infra technology can result in infringement of privacy as new devices and media are being developed. However, with the current legal system, it is inevitable to face certain dead zones due to the fast development of technology that 
outruns the development of new regulation. Thus, institution of neutral regulation that can protect privacy regardless of emergence of new technology is required.

Lastly, clear legal standard should be provided on the limitation of personal data collection. Privacy infringement can also occur in the u-City environment by service providers' profiling of wide range of personal data through various channels. Therefore, legal framework to regulate profiling personal data should be introduced.

\subsection{Technical Suggestions in Building a Safe u-City}

Another challenge that should be tackled in building safe u-city besides shaping up new legal and institutional framework is developing new technologies to protect privacy. Alongside the already existing PETs (Privacy Enhancing Technologies) such as P3P, Privacy Policy Statements Generators, cookie control, SW encryption, and anonymity technology, advanced technologies for the u-city environment need to be developed. Continuous R\&D efforts for the development of advanced technologies (such as technologies on encryption to prevent information leakage, safe information processing in the wire and wireless network, PETs on data transfer and containment, authentication, and personal identification management) are called for.

In addition, the protection of personal locational and circumstantial information should be ensured in the u-City environment by realizing the integrated PETs that can protect anonymity in addition to the current ID management technology. Anonymity, tracking and traffic analysis are the fields which have not deserved a due consideration yet, but in the u-City environment, these areas are the essential requirement that need to be ensured to protect personal data.

Also, as the existing infra technologies keep developing and new technologies keep appearing, seamless R\&D efforts to deal with the novel technological challenge is required to build a safe u-city.

\section{Conclusion}

Different from traditional cities, the u-City is the forthcoming intelligent city, engrafting highly sophisticated ubiquitous IT technologies such as sensing \& RFID technologies, context-aware technology, mobile network technology, and so on. However, due to these advancements, the u-City raises many new problems relating to private information. Because the concept of the u-City is quite new, not only is there a lack of related research in general, but studies on the implication of the u-City on privacy protection are also scarce.

Thus this paper has carefully observed several types of privacy issues that may arise under the environment of the u-City in comparison with traditional cities. It also has suggested legal, institutional and technical complementary measures to solve privacy problems in the u-City. In terms of legal and institutional aspects, measures should be taken to provide previous notification and ask for the consent of the data subject to enhance privacy protection in the u-City. Also, the necessities of taking measures relating to guarantee of anonymity for sensitive information, establishment of value-neutral laws to prepare for new technologies and laws on profiling of 
personal data are explained in the paper. For technical aspects, the paper has proposed a future direction of how the PETs should develop under the environment of the u-City.

In addition, for establishing the safe u-City, raising individual awareness for protection of private information, expanding self-regulatory movements, and training specialists for personal data protection should be pursued along with legal, institutional and technical measures. Furthermore, overemphasis on privacy protection may have a danger of undermining the overall efforts to achieve the u-City. Therefore, achieving a stable equilibrium of private information protection and a wise use of personal data for informatization should always be at our focal interest.

\section{References}

1. Kim, M. S., New City in the Era of Ubiquitous: u-City, Korean National Assembly Library Press, Vol. 325 (2006) 40-45

2. Ishida, T., Isbister, K. (ed.): Digital Cities: Experiences, Technologies and Future Perspectives, Lecture Notes in Computer Science, Vol. 1765. Springer-Verlag (2000) 350-363

3. Park, J. S., Lim, H. B., The Concept of u-City and Business Strategy, Communication Market, Korea Telecom Management Institute, Vol. 59 (2005) 3-4

4. Solove, D. J., A Taxonomy of Privacy, University of Pennsylvania Law Review, Vol. 154, No. 3 (2006) 477-560, Available at SSRN: http://ssrn.com/abstract=667622.

5. Kim, T. J., Lee, S. W., Lee, E. Y., Privacy Engineering in ubiComp, ICCSA 2005, Lecture Notes in Computer Science, Vol. 3482 (2005) 1279-1288

6. US: US Safe Harbor Privacy Principles (2000)

7. OECD, Guidelines Governing the Protection of Privacy and Transborder Flows of Personal Data (1980)

8. UN, Guidelines for the Regulation of Computerized Personal Data Files (1990)

9. EU, Directive 95/46/EC of the European Parliament and of the Council of 24 October 1995 on the Protection of Individuals with regard to the Processing of Personal Data and on the Free Movement of Such Data (1995)

10. APEC, APEC Privacy Framework (2004) 